\title{
Rotatostereoradiography: A New Radiodiagnostic Method
}

\section{-Development of a New Three-dimensional Radiodiagnostic Device and Evaluation in Neurosurgical Clinics-}

\author{
Michinori OTTOMO, Norihiko BASUGI*, Hajime HANDA**, \\ Takashi TANIGUCHI $^{* * *}$, Takashi IWABUCHI ${ }^{\dagger}$ and Mitsuo KOSAKA $^{\dagger \dagger}$ \\ Department of Neurological Surgery, Aomori Rosai Hospital, Hachinohe, Aomori; \\ *Department of Neurosurgery, Kantoh Rosai Hospital, Kawasaki, Kanagawa; \\ **Hamamatsu Rosai Hospital, Hamamatsu, Shizuoka; ***Labour Welfare Corporation, \\ Tokyo; ${ }^{\dagger}$ Department of Neurosurgery, Hirosaki University School of Medicine, \\ Hirosaki, Aomori; ${ }^{\dagger}$ Medical System Division, Shimadzu Corporation, Kyoto
}

\begin{abstract}
The rotatostereoradiographic device uses an $\mathrm{x}$-ray tube coupled with an image intensifier rotating through a $180^{\circ}$ arc in 2.25 seconds. The rapidly rotating $\mathrm{x}$-ray tube allows $180^{\circ}$-are angiograms to be obtained with a single injection of contrast medium. Subtracted fluoroscopic angiograms can be viewed immediately after injection of the contrast medium with digital recording. These three-dimensional images are displayed on side-by-side monitors stereoscopically.

The mortality and morbidity of subarachnoid hemorrhage can only be greatly reduced by surgical treatment of unruptured aneurysms and arteriovenous malformations detected by a wide survey of subarachnoid hemorrhage. Such a wide survey would be possible utilizing intra-arterial digital subtraction angiography via the ascending aorta and this new three-dimensional radiodiagnostic method. A fluoroscopic device must be used to allow easier manipulation of the catheter from the axillary or brachial artery.
\end{abstract}

Key words: angiography, anatomical study, diagnostic imaging, radiography, stereoangiography

\section{Introduction}

The discovery of the x-ray in 1895 allowed the threedimensional human body to be presented as twodimensional perspective images. Radiological diagnosis of specific lesions was based on these twodimensional images of three-dimensional organs. This required special training for radiologists in the organ systems. Recently, coronal and sagittal tomographic images of organs have been obtained easily by computed tomography (CT) and magnetic resonance (MR) imaging. ${ }^{5)}$ Image-processing technology, however, can only manipulate the original image data. Therefore, if an aneurysm neck is not

Received September 30, 1988; Accepted September 12, 1989 clearly visualized on the original angiograms, an aneurysm neck appearing in an image formed by image-processing technology ${ }^{16)}$ may not in fact exist. Although digital subtraction angiography (DSA) achieves excellent image contrast resolution, the precise information needed on the afferent and efferent vessels of the aneurysm or arteriovenous malformation (AVM) cannot be obtained. Computer technology was therefore used both for subtraction and three-dimensional display of the images but not for any reforming technique in our rotatostereoradiographic (RSRG) device. The RSRG equipment uses a real-time, $180^{\circ}$-arc, three-dimensional display of the afferent and efferent vessels of an aneurysm or AVM with a single injection of contrast medium, to provide detailed information. 


\section{Materials and Methods}

\section{Gantry and x-ray high-voltage generator}

Gantry (AR-10, certificate number 62B1194, ROTATO-360 Type 87.00; Shimadzu Corp., Kyoto) (Fig. 1): A large-focus, 1-mm x-ray tube (Circlex 1P39AK; Shimadzu Corp.) and a 9-inch metal, highcontrast image intensifier (IA-9T-1-2W; Shimadzu Corp.) (I.I.) to convert $\mathrm{x}$-rays into light signals are used. An X-ray fluoroscope (AX-2020P; Matsushita Communication Industrical Co., Ltd., Tokyo) to convert light signals into an image and a diode gun plumbicon tube with low after-image are also used.

The $x$-ray tube coupled with the I.I, rotates through a $180^{\circ}$ are in 2.25 seconds (high speed) or

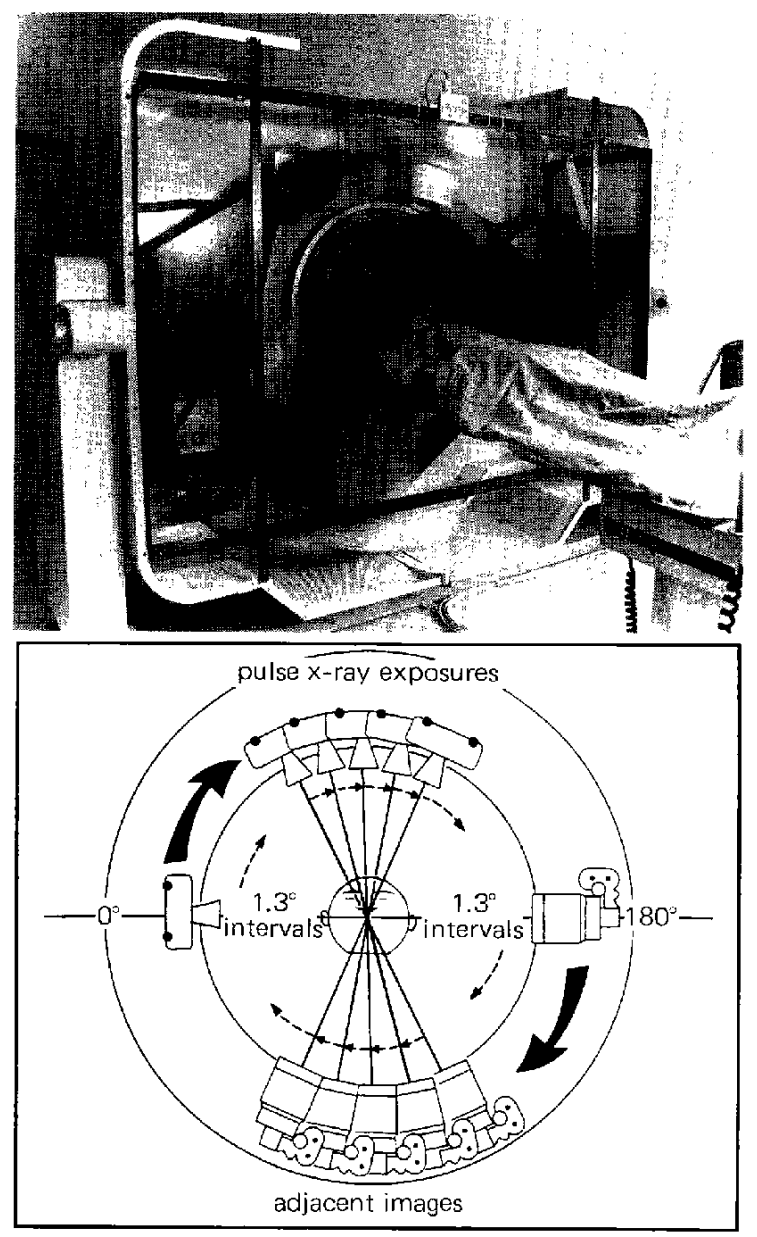

Fig. 1 RSRG device (AR-10). upper: The gantry carrying the opposed $\mathrm{x}$-ray tube and I.I., which rotate in 2.25 seconds through a $180^{\circ}$ arc. lower: Schematic illustration, showing the relationship between the pulse $x$-ray exposures and adjacent images changing the rotation interval by $1.3^{\circ}\left(1^{\circ} 18^{\prime}\right)$.
4.50 seconds (slow speed). The distance between the $\mathrm{X}$-ray tube and the I.I. is $100 \mathrm{~cm}$, with $63 \mathrm{~cm}$ between the focal point and the $x$-ray rotation center, utilizing the $70-\mathrm{cm}$ diameter gantry aperture. Tilted angles of $\pm 20^{\circ}$ are provided to take angiograms in either the Towne or Waters views.

Fluoroscopy provides magnified images of organs, because the magnification is greater when the subject is closer to the $\mathrm{x}$-ray tube. The $\mathrm{x}$-ray tube must be rotated on the operative approach side to maintain the viewer's orientation. In practice, an over-table tube rotation is required for a pterional approach such as a frontotemporal craniotomy, and an under-table tube rotation must be used for a posterior approach such as a suboccipital craniectomy.

$\mathbf{X}$-ray generator: In order to use this method for whole body investigation, a high-voltage $\mathrm{x}$-ray generator with constant potential controlled by a microprocessor (HD150G-60; Shimadzu Corp.) is employed.

Cinematography: A 35-mm cine system (Arritechno 35R90; Arnold \& Richter Cine-Technik, Munich, Germany) is used for cine angiography. The images are viewed stereoscopically using a $35-\mathrm{mm}$ cine film stereoprojector. ${ }^{8)}$

II. Digital fluoroscopic unit (prototype, DF-150 Type 87.00; Shimadzu Corp.)

Digital memory device: $\mathrm{X}$-ray fluoroscope signals are converted into digital information by an 8-bit A/ $\mathrm{D}$ converter and recorded by a 96-megabyte digital memory device (SDM-96; Cybernetics Technology, Co., Ltd., Tokyo). Each of 136 field images for plain radiograms and angiograms requires $512 \times 512 \times 8$ bits.

$X$-ray exposure: $\mathrm{X}$-rays are exposed in two modes. In "rotato mode," the images are obtained with the x-ray tube rotating. In "serial mode," the images are taken with the x-ray tube fixed as for conventional angiography. Images are photographed at 60 fields per second ( $\mathrm{f} / \mathrm{s}$ ) ( 30 frames per second) for 5 seconds, which gives the best resolution for fluoroscopy. To lengthen the photographing time, the number of x-ray exposures can be reduced, for example to $30 \mathrm{f} / \mathrm{s}$ for 10 seconds or $20 \mathrm{f} / \mathrm{s}$ for 15 seconds. To use the rotato mode for intravenous DSA, the circulating time for the contrast medium from the vena cava to the cerebrum must previously be evaluated, because the x-rays must be exposed just as the contrast medium reaches the cerebrum. Ten-ml contrast medium is required for this purpose using the serial mode.

In the rotato mode, plain radiograms are initially obtained. Angiography is then performed during 
the injection of contrast medium. Digital subtraction of these images can subsequently be performed. In the final process, the position of a "doll's head" is placed in one of eight areas in the center of the monitor.

Display monitors: The rapid rotational display of linear subjects provides three-dimensional depth information. A single " $3-D$ monitor" is provided for this.

Every consecutive pair of images obtained by the rotato mode makes a stereo-pair, because of the small difference in $\mathrm{x}$-ray exposure position (Fig. 1 lower). Consequently, the images displayed on sideby-side monitors, e.g. 1 (left) and 2 (right), 2 (left) and 3 (right), 3 (left) and 4 (right), etc., can be viewed stereoscopically. Two side-by-side "stereo display monitors" with a stereoviewer (S Type Stereomirror V; Seiko Rika Medical Electronic Co., Ltd., Osaka) are therefore provided. A hood between the stereoviewer and the stereo monitors simplifies the stereoscopic viewing of images. A central plate is placed within the hood, so a single screen is viewed with each eye (left monitor by left eye, right monitor by right eye).

Display methods: In "rotational mode," one-way rotating images are repeatedly displayed. The images are therefore viewed in an endless cycle by linking the first portion of the cine film to the last.

In "oscillating mode," alternate right and left rotational displays of the images are viewed. The rotating images are consequently viewed like a fan.

In "progressive oscillating mode," one-step advance is performed during one oscillating display. The advance is similar to "four steps forward, three steps backward." "9) Although the starting position angle and the swing width of rotating images can be chosen within $180^{\circ}, 0^{\circ}$ and $80^{\circ}$ are initially provided.

"Still mode," a pause in the rotating images, can easily be obtained by pushing the "space" key. In this mode, one-frame forward or backward movement of the images is performed by pushing the " 1 " or " 3 " key. Images can be photographed on film. Restart of image rotation is obtained by pushing the "space" key again.

Image recording: The images are recorded on a laser disc (Laser Disc Subsystem WM-S050; Toshiba Corp., Tokyo). The oscillating images are also recorded on video tape for clinical use. The paired stereo images can be photographed on imaging film using a multi-camera (FIM3535AG; Fuji Medical Systems Co., Ltd., Tokyo). Such photographed images can be viewed stereoscopically on the ward.

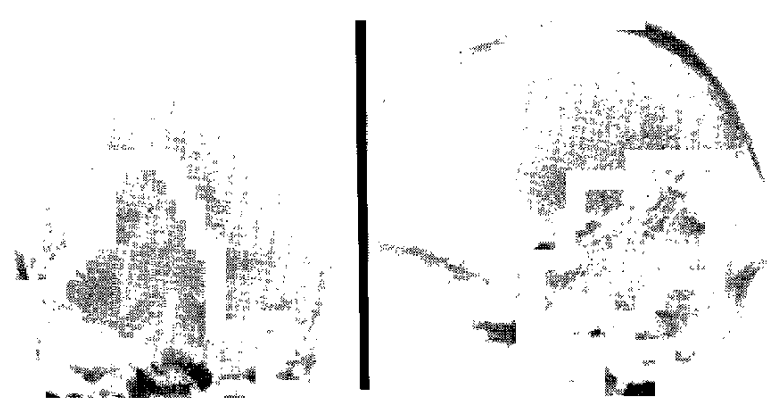

Fig. 2 Conventional transaxillary retrograde left vertebral angiograms of a 19-year-old female with an AVM in the right occipital lobe. These two-dimensional angiograms were obtained using a different injection of contrast medium for each image. left: Anteroposterior view, right: lateral view.

\section{Results}

Conventional transaxillary retrograde left vertebral angiograms of an AVM are shown in Fig. 2. With this method, contrast medium injection was required for each anteroposterior, lateral, oblique, or contraoblique projection. The left vertebral rotatostereoangiograms of the same patient are shown in Fig. 3. Since $180^{\circ}$-arc angiograms are obtained using a single injection of contrast medium, it is possible to obtain the precise relationship between the afferent and efferent vessels of the AVM.

Simultaneous three-phase (arterial, capillary, and venous) cerebral angiograms of the same patient are shown in Fig. 4. The relationship between the nidus and the afferent and efferent vessels of the AVM can be clearly seen in these stereoscopic pairs of bilateral posteroanterior oblique views. The angiograms are viewed through faint staining of cerebellar architecture.

A four-vessel study (intra-arterial DSA) of the same patient is shown in Fig. 5. Although the bilateral middle cerebral arteries are viewed closely together in the lateral view, there is no difficulty in defining the precise anatomical relationship due to the stereoscopic display. In spite of the blurred shadows of the paused images in these figures, images displayed at $60 \mathrm{f} / \mathrm{s}$ are clearly viewed on the video of the fluoroscope.

Rotatostereoradiograms of the venous phase easily visualize obstructions of the cavernous sinus or jugular foramen. These images were obtained by stopping contrast medium injection 3 seconds before $\mathrm{x}$-ray tube rotation. The left carotid venous-phase angiograms of a 62-year-old female with Tolosa- 


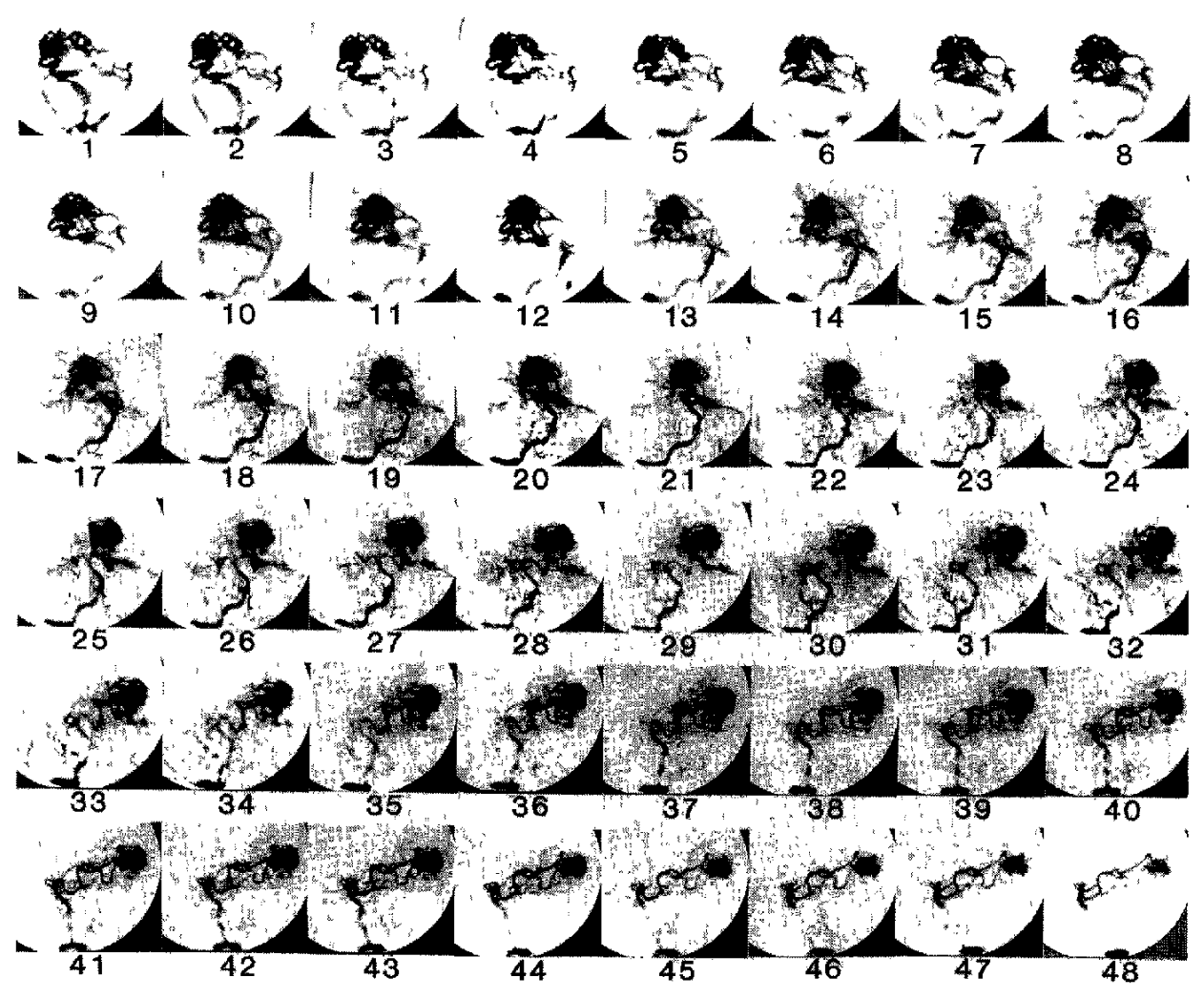

Fig. 3 Left vertebral rotatostereoangiograms of the same patient as in Fig. 2 using Seldinger's technique. Although the right and left relationship is reversed from Fig. 2 because the under-table tube rotation was used, consecutive paired angiograms are viewed stereoscopically from right lateral (1 and 2), through posteroanterior (23 and 24 ), to left lateral (47 and 48 ) projections.

Hunt syndrome are shown in Fig. 6.

Simultaneous two-phase (arterial and venous) carotid angiograms ${ }^{3,9)}$ using two injections of contrast medium with a 2 -second interval can demonstrate a chronic subdural hematoma with cortical vessels or a vascular tumor with its afferent and efferent vessels clearly.

Although clear cervical arteriograms can be obtained by intravenous DSA from the superior or inferior vena cava, intra-arterial DSA from the ascending aorta are superior as shown in Fig. 7.

\section{Discussion}

Stereoradiography obtains paired stereo images by rotating the head from the midline to medial aspect of the eyebrows during $x$-ray exposure ${ }^{14)}$ or by rotating the $x$-ray tube in $5^{\circ}$ increments. ${ }^{13)}$ In 1980 , we reported a new diagnostic method called "serial rotatostereography," in which the head is rotated from the anteroposterior $\left(0^{\circ}\right)$ to lateral $\left(90^{\circ}\right)$ views in small increments, each requiring separate injections of contrast medium. ${ }^{11)}$ This method obtains con-

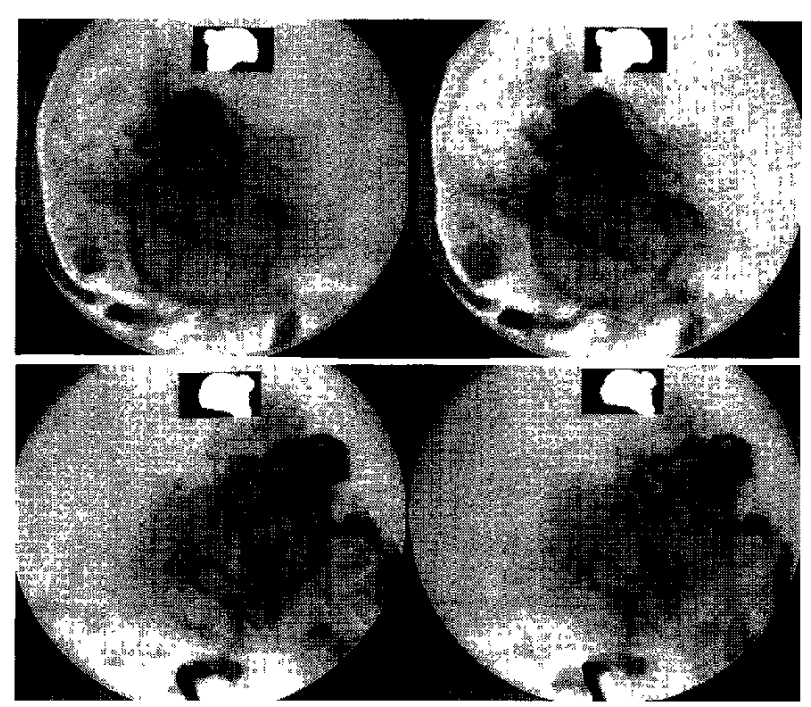

Fig. 4 Simultaneous three-phase carotid angiograms of the same patient as in Figs. 2 and 3. A total of $20 \mathrm{ml}$ contrast medium was injected at $3 \mathrm{ml} /$ sec with initiation of tube rotation 4 seconds post-injection. upper: Oblique view, lower: contra-oblique view. 


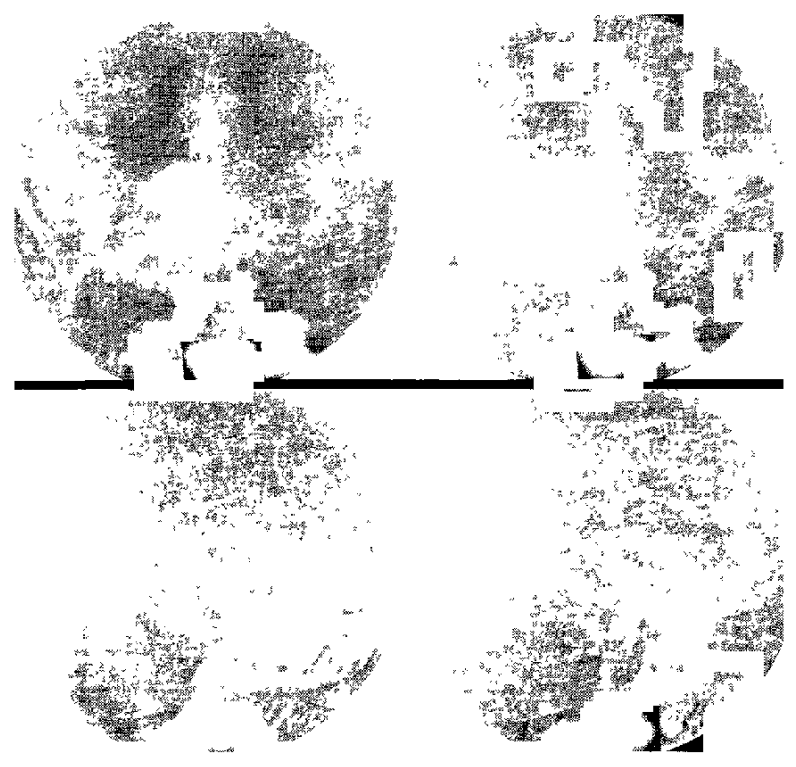

Fig. 5 Four-vessel study (intra-arterial DSA) of the same patient as in Figs. 2-4. Because the overtable tube rotation was used, the right and left relationship is reversed from Figs. 3 and 4. Stereoscopic pairs of anteroposterior (upper) and lateral (lower) views were obtained using a single injection of $50 \mathrm{ml}$ contrast medium at $18 \mathrm{ml} / \mathrm{sec}$ in the ascending aorta.

tinuous stereoscopic images from anteroposterior to lateral views using films 1 and 2,2 and 3 , etc. ${ }^{6}$ However, multiple injections of contrast medium are undesirable for clinical use. ${ }^{2)}$ We initially intended to use cine angiography and computer technology to decrease injection times. ${ }^{11}$ The application of cine angiography and development of a cine film stereoprojector were achieved in $1983 .{ }^{7)}$ As a result, stereoscopic cerebral angiograms could be viewed through a $180^{\circ}$ arc while requiring a single contrast medium injection, by using a specially designed "Stereotom" and "cine film stereoprojector." stereoscopic images could be viewed easily utilizing a hood with a central wall separating the left and right visual fields. ${ }^{9}$

The time required for obtaining cinematographic film is too long for a real-time stereo display, so a video disc recorder was used. The possible clinical use of a video disc recorder was studied using two video memories in $1986 .{ }^{(0)}$ The following study investigated DSA using a cardiovascular analyzer ACIA-320 in 1987.9) The resolution of images by this method was inferior to the cine method because a 525-line monitor was used. Another limitation was the requirement for two rotations of the $\mathrm{x}$-ray tube through a $180^{\circ}$ arc, one for plain radiograms and

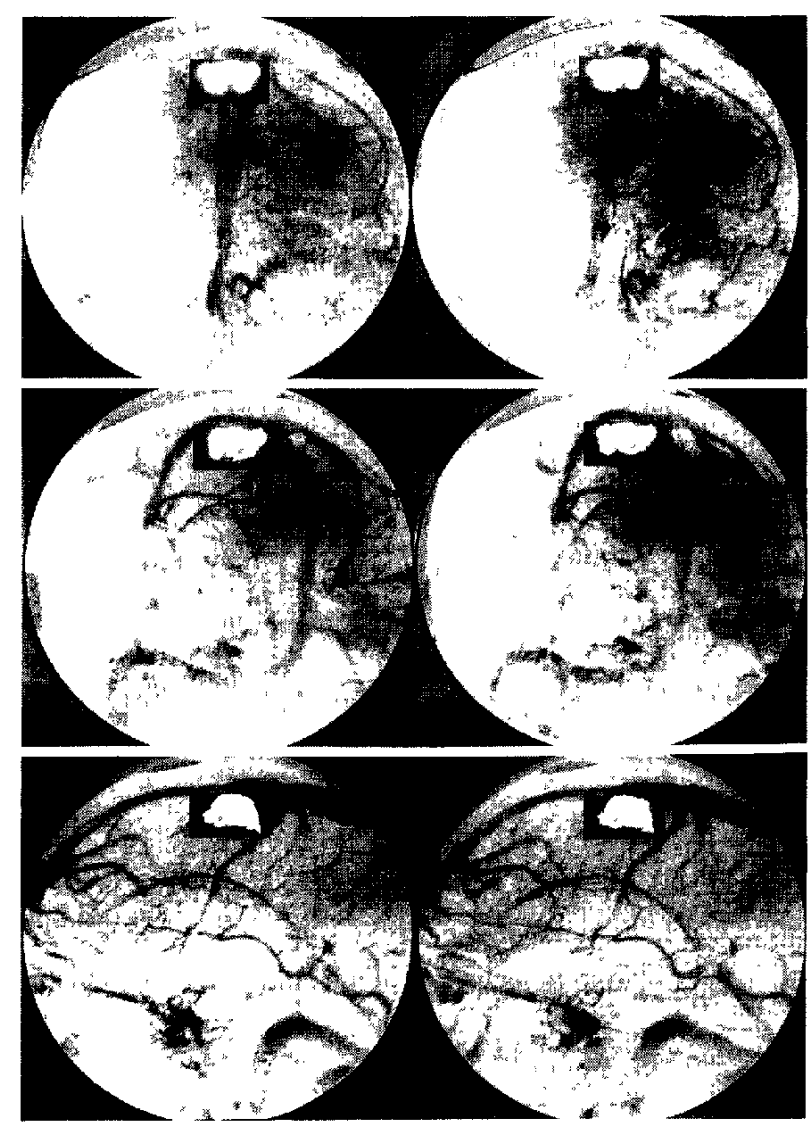

Fig. 6 Left carotid venous-phase angiograms of a 62year-old female with Tolosa-Hunt syndrome. The relationship between the left cavernous sinus and the afferent and efferent venous circulation is clearly visualized by these stereoscopic pairs of anteroposterior (upper), oblique (middle), and lateral (lower) views. A disturbance of the venous flow through the cavernous sinus is clearly recognized.

the other for angiograms. These problems resulted in failure in clinical use. However, the $60-\mathrm{f} / \mathrm{s}$ fluoroscopic display provided excellent resolution. In addition, the blurring caused by image subtraction by the rotating $\mathrm{x}$-ray tube was minimized because of the stereoscopic display capability. ${ }^{\text {9) }}$ As a consequence of these changes, the new radiodiagnostic device for RSRG was developed.

In the RSRG device, the magnification is $1.6 \times$ (100/63). The side-by-side monitors display every other field image, e.g. 1 (left) and 3 (right), 2 (left) and 4 (right), 3 (left) and 5 (right), and so on, to maintain an equal magnification in three dimensions. The focus separation between these images is $29 \mathrm{~mm}[(2 \times 3.14 \times 630) \times(180 / 360) \times(2 / 136)]$. Takahashi et al. ${ }^{15)}$ employed a $35-40-\mathrm{mm}$ tube shift 

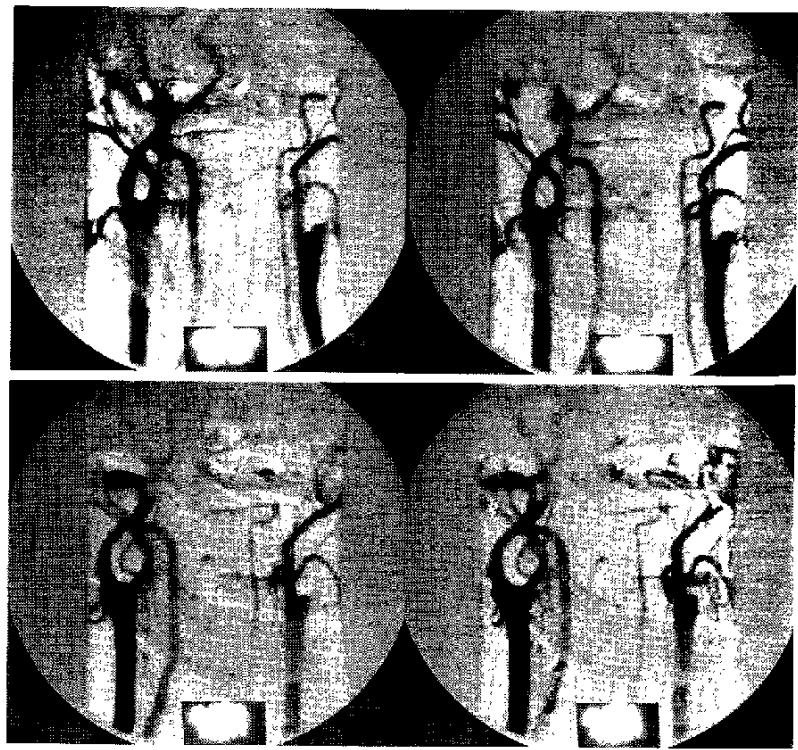

Fig. 7 Cervical four-vessel study (intra-arterial DSA) using a single injection of contrast medium in the ascending aorta of a 50-year-old male. Obstruction of the left internal carotid artery and slight stenosis of the right proximal internal carotid artery are clearly visualized with the anatomical relationship between the carotid and vertebral arteries in these stereoscopic pairs of anteroposterior (upper) and right oblique (lower) views.

with a $90-\mathrm{cm}$ distance between the focus and the film for $2 \times$ magnification radiography. Although the 29$\mathrm{mm}$ focus separation in $1.6 \times$ magnification radiography is smaller than the $35-\mathrm{mm}$ tube shift in $2 \times$ magnification, an equal magnification in three dimensions could be obtained utilizing a longer viewing distance using a stereomirror with a hood in this system. The angiograms in Figs. 3-7 demonstrate more blurring shadow than conventional DSA because a large, 1-mm focus $\mathrm{x}$-ray tube is required for cine pulse exposures. In addition, it is impossible to use computer software technology to average consecutive digital images due to the different rotationangle exposures. These problems can be easily resolved by using an $x$-ray tube with a small focus and large heat strage capacity and applying computer software for rotating MR angiograms.

The whole concept of diagnosis based on the relative differences in gray shades on $\mathrm{x}$-ray film was basically changed by the development of CT in 1972 . CT scans easily demonstrate intracerebral hemorrhage, infarction, and tumor by image-processing technology, utilizing a computer and television monitor. Such digitized image technology has been applied to every radiodiagnostic machine, implying that it might be possible to show every pathological lesion in the body by image-processing technology only. There are, however, limits to reforming technology especially in cerebral angiography even using the DSA technique. These problems were easily resolved by the $x$-ray tube coupled with an I.I. rotating around the body. Recently, the transmission of images by satellite signals was required. Because the rotating image display of $60 \mathrm{f} / \mathrm{s}$ has excellent resolution using fluoroscopy with three-dimensional depth information, this method makes it possible to easily understand the relationship between afferent and efferent vessels of aneurysms by viewing a television monitor.

The term "three-dimensional display" is frequently used since the widespread use of computer graphics. ${ }^{16)}$ This is not the same as "stereoscopic display," which has been in use since 1898. "We previously proposed the "classical stereo display method" (using two cathode-ray tubes and a stereoviewer) and the "new stereo display method" (using a single cathode-ray tube), which displayed images in a semirotating fashion similar to a oscillating fan head. ${ }^{10)}$ Stereoscopic display depends fundamentally on paired images taken from two slightly different angles. There are three stereoscopic display methods using paired images: 1) viewed through a stereoviewer on different side-by-side screens; 2) viewed through a green, red, or polarized glass on a single screen; and 3) viewed through a glass with a PLZT electro-optic shutter on a single monitor. ${ }^{12)}$ With all these methods, stereoscopic viewing of the images requires both right and left eyes. In addition, there are two more three-dimensional display methods: 4) showing a single still image with depth information using the techniques of shape representation, hidden line and surface, co-ordinate transformation, shading and texture mapping, and ray tracing ${ }^{16)}$; and 5) showing three-dimensional images on a single screen or monitor with movement. In cine or video, three-dimensional depth information is obtained by adding the time component ( $Z$ axis) to the two-dimensional $X-Y$ axes of a single screen space. ${ }^{10)}$ A three-dimensional device must therefore be capable of showing images using one of the methods 1) to 3) and also 4) or 5).

Logically then, one can view three-dimensional images utilizing cine or video, but cannot view these images stereoscopically because of the lack of paired stereo images. Our "classical" and "new" stereo display methods could be renamed the "stereoscopic" and the "three-dimensional" displays, respectively. Recently, the brainstem and cerebral cortex 
have been simultaneously displayed by CT and MR imaging, similar to showing an apple core by removing a quarter of the whole. Although these images are viewed stereoscopically, these techniques are classified as three-dimensional display because no paired stereo images are used. ${ }^{4}$ On the other hand, our RSRG device could be called a "threedimensional radiodiagnostic device," because of the stereoscopic display using two stereo monitors and three-dimensional display using rotational movement of the images (methods 1 and 5).

\section{Acknowledgments}

We are grateful to Prof. Shoichi D. Takekawa, Department of Radiology, and the staff of the Department of Neurosurgery, Hirosaki University School of Medicine; Prof. Toshimitsu Musha and Prof. Takeshi Agui, Tokyo Institute of Technology; Prof. Kenji Hiwatashi, the Faculty of Science and Engineering, Meisei University; Mr. Motohisa Tsuda, Shimadzu Science Foundation; Mr. Akiharu Eto and the late Shigeru Sutoh, Eto Shoji Co., Ltd.; Mr. Takeshi Nakanishi and his staff, Shimadzu Corp.; Mr. Masafumi Fujimoto and his staff, Cybernetics Technology Co., Ltd.; Mr. Shoji Tenman, Seiko Rika Medical Electronic Co., Ltd.; and Mr. Masao Sawada and his staff, Department of Radiological Technology, Aomori Rosai Hospital; for their kind help with this study.

The Labour Welfare Corporation has founded 38 Rosai Hospitals throughout Japan to ensure the improved health and living conditions for both workers and their families. The Corporation has developed a RSRG device to achieve a greater reduction in mortality and morbidity of vascular diseases by early diagnosis and treatment. We are grateful to Yasuo Katabira, Director, Hajime Fujita, Vice-director, Yoshinori Miyatsu, Secretary-general, and the staff of Aomori Rosai Hospital, and the staff of the headquarters of the Labour Welfare Corporation dealing with this device.

Parts of this paper were presented at the 46th Annual Meeting of the Japan Neurosurgical Society, held in Tokyo, October, 1987; the 35th Annual Meeting of Japanese Traumatology and Occupational Medicine, in Tokyo, October, 1987; the 73rd Annual Meeting of Radiological Society of North America, in Chicago, November-December, 1987; the 17th Annual Meeting of the Japanese Neuroradiological Society, in Tsukuba, February, 1988; the 47th Annual Meeting of Japan Radiological Society, in Tokyo, March-April, 1988; and the 12th Annual Meeting of the Joint Meeting of the Four
Neurosurgical Conferences in North Japan, in Toyama, June, 1988.

\section{References}

1) Davidson JM: Remarks of the value of stereoscopic photography and skiagraphy: Records of clinical and pathological appearances. Brit Med $J$ 3: 1669-1671, 1898

2) Fujino Y: Opinions of the chairman. Nippon Igaku Hoshasen Gakkai Zasshi 42: 81, 1982 (in Japanese)

3) Iwabuchi $T$, Ito $Z$, Suzuki J: A technique of simultaneous two phase carotid angiography. Amer $J$ Roentgen 101: 116-120, 1967

4) Maeda T: The present state of PACS. Medical Imaging Technology 6: 2-8, 1988 (in Japanese)

5) Onoe M: Usefulness of three-dimensional images in medicine. Saishin Igaku 41: 15-20, 1986 (in Japanese)

6) Ottomo M: Serial rotatostereography - a new diagnostic method, I. Application to cerebral angiography and to ventriculography. Nippon Saigai Igakkai Kaishi 31: 592-603, 1983 (in Japanese)

7) Ottomo M: Serial rotatostereography - a new diagnostic method, II. Experimental study for cineangiography utilizing new method for stereoviewing cineangiograms with clinical application. Nippon Igaku Hoshasen Gakkai Zasshi 43: 1036-1043, 1983 (in Japanese)

8) Ottomo M: Serial rotatostereography - a new diagnostic method (part III). Manufacture of Stereotom Type I and stereoprojector for cine film. Neurol Med Chir (Tokyo) 24: 840-847, 1984 (in Japanese)

9) Ottomo M, Minakami S, Nakano M, Sugawara K, Sutoh S, Kosaka M: Application of digital subtraction angiography for serial rotatostereoradiography utilizing a cardiovascular image analyzer ACIA-320. Eizo Joho Medical 19: 751-757, 1987 (in Japanese)

10) Ottomo M, Nakanishi T: Serial rotatostereography a new diagnostic method. Development of real time display method: Continuous semi-rotation stereo display method. Neurol Med Chir (Tokyo) 26: 759764, 1986 (in Japanese)

11) Ottomo $M$, Saitoh $K$, Tsubakisaka $H$, Nakaoka $T$ : A new method of stereoangiography. Rotating serialstereoangiography. Neurol Med Chir (Tokyo) 20 [Suppl]: 178-179, 1980 (in Japanese)

12) Roese JA, Turner AF: Single monitor stereoradiological television system using PLZT electrooptic shutters. Radiology 121: 743-744, 1976

13) Shibata T, Ito A, Miyaji S, Kajita Y, Mori Y: Rotational stereoscopic cerebral angiography. Neurol Med Chir (Tokyo) 28: 871-874, 1988 (in Japanese)

14) Skelly GA, Mansour G: A simple method of stereoscopic angiography with particular reference to the posterior fossa. Amer J Roentgen 118: 690-694, 1973

15) Takahashi M, Ozawa $Y$, Takemoto H: Focal-spot separation in stereoscopic magnification radiogra- 
phy. Radiology 140: 227-229, 1981

16) Toriwaki J: Computer graphics - present and future. Saishin Igaku 41: 5-14, 1986 (in Japanese)
Address reprint requests to: M. Ottomo, M.D., Department of Neurological Surgery, Aomori Rosai Hospital, 1 Minamigaoka, Shirogane-cho, Hachinohe, Aomori 031, Japan. 